\title{
Efeito da goma guar parcialmente hidrolisada no metabolismo de lipídeos e na aterogênese de camundongos
}

\author{
Effect of partially hydrolyzed guar gum on \\ lipid metabolism and atherogenesis of mice
}

\author{
Luciana Rodrigues FERNANDES' \\ Marilene Diniz XISTO' \\ Michele Groenner PENNA' \\ Izabela Mascarenhas MATOSINHOS ${ }^{1}$ \\ Matheus Calab LEAL ${ }^{1}$ \\ Luciane Rodrigues PORTUGAL ${ }^{1}$ \\ Jacqueline Isaura Alvarez LEITE'
}

\section{$\|$} ORIGINAL | ORIGINAL . .

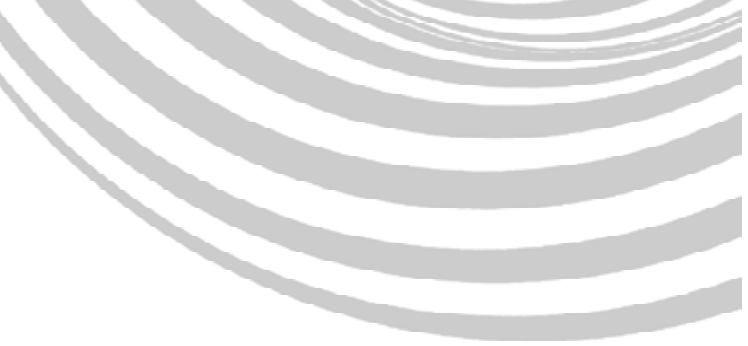




\section{Conclusão}

Os resultados sugerem que a goma guar parcialmente hidrolisada pode reduzir a aterosclerose associada ao Diabetes Mellitus tipo 1.

Termos de indexação: aterosclerose; colesterol; Diabetes Mellitus; fibras na dieta.

\section{A B S T R A C T}

\section{Objective}

The objective of this study was to observe the effects of partially hydrolyzed guar gum on cholesterol metabolism and atherosclerosis in the aorta of euglycemic and streptozotocin-induced hyperglycemic $L D L$ receptor deficient mice.

\section{Methods}

Thirty six LDL receptor deficient mice were divided into 4 groups of 9 animals: euglycemic groups fed on hypercholesterolemic diet without or supplemented with $7.5 \%$ of partially hydrolyzed guar gum and streptozotocin-induced hyperglycemic groups also fed an atherogenic diet without or supplemented with 7.5\% of partially hydrolyzed guar gum. After 4 weeks of experiment, food intake, body weight, glycemia, blood and liver cholesterol and atherosclerotic lesion in the aorta were determined.

\section{Results}

The results showed that partially hydrolyzed guar gum induced an increase in blood and liver cholesterol in euglycemic mice when compared with euglycemic control groups at the end of the experiment. On the other hand, although not affecting plasma cholesterol, hyperglycemic mice supplemented with partially hydrolyzed guar gum had the lesion area in the aorta significantly reduced. In hyperglycemic animals, plasma cholesterol did not decrease significantly but the lesion area in the aorta did.

\section{Conclusion}

The present study suggests that partially hydrolyzed guar gum can reduce the development of atherosclerosis associated with type 1 diabetes mellitus.

Indexing terms: atherosclerosis; cholesterol; Diabetes Mellitus; dietary fiber.

\section{N T R O D U ÇÃ O}

A doença cardiovascular é a principal causa de mortalidade no mundo. Suas origens são diversas, sendo que o aumento do colesterol na lipoproteína de baixa densidade (LDL) e/ou sua oxidação, são condições essenciais para a aceleração do processo. Entre os fatores de risco para aterosclerose estão hipertensão arterial, tabagismo, dislipidemias, história familiar e idade. o Diabetes Mellitus é considerado um importante fator de risco independente para doença arterial coronária, sendo caracterizado pelo III National Cholesterol Education Program (NCEP) ${ }^{1}$ como um equivalente de doença coronariana, dada a sua alta correlação com esta doença cardiovascular. A associação entre doença aterosclerótica e Diabetes Mellitus pode ser atribuída a fatores como: obesidade, aumento de radicais livres, hipertensão arterial sistêmica, níveis elevados de fibrinogênio² dislipidemias, entre outros ${ }^{3}$.

A causa das hiperlipidemias nos pacientes diabéticos envolve a redução da insulinemia que, por sua vez, reduz a atividade da lipase de lipoproteína, enzima responsável pela hidrólise de triacilgliceróis de quilomícrons e lipoproteína de muito baixa densidade (VLDL), levando à hipertrigliceridemia e à redução do fator de proteção lipoproteína de alta densidade (HDL), formada a partir dos remanescentes dessas lipoproteínas. A redução de insulina também acelera a lipólise no tecido adiposo, promovendo importante liberação de ácidos graxos livres, que funcionam como substrato para a produção de VLDL no hepatócito 4 . 
A lipoproteína de baixa densidade (LDL) é a principal transportadora de colesterol para os tecidos periféricos. Essa lipoproteína liga-se às células pelo receptor de $L D L$, que pode regular a concentração intracelular de colesterol. Altas concentrações de colesterol intracelular regulam negativamente a expressão do ácido ribonucléico (RNA) mensageiro do receptor $L D L$ e da enzima chave na síntese de colesterol (3-Hidroxi-3-metil-glutaril CoA redutase).

A deleção do gene que expressa o receptor para LDL deu origem ao camundongo com deficiência para o gene que expressa o receptor de $\operatorname{LDL}\left(\mathrm{LDLr}^{--}\right)$. Esse animal possui aumento significante nos níveis sanguíneos das lipoproteínas aterogênicas LDL e lipoproteína de densidade intermediária (IDL), apresentando um quadro de hipercolesterolemia moderada semelhante àquele descrito em humanos ${ }^{5}$. Esse modelo animal tem sido extensivamente utilizado na investigação de mecanismos envolvidos no desenvolvimento da aterosclerose, como também em ensaios medicamentosos $^{6}$.

A hipercolesterolemia e a formação das lesões ateroscleróticas nos camundongos LDLr-1ocorrem pelo uso de uma dieta comercial, ou podem facilmente ser aceleradas quando se tem um suplemento contendo gordura e colesterol. Dessa forma, esses animais fornecem um excelente modelo para avaliação dos eventos aterogênicos iniciais. As lesões ateroscleróticas nesses animais, uma vez caracterizadas, apresentam-se semelhantes àquelas encontradas em humanos?.

A fibra alimentar consiste em carboidratos não digeríveis e lignina, intrínsecos de plantas intactas. Além da ingestão pela dieta, o aporte diário de fibras pode ser alcançado pelo uso de suplementos ou isolados de fibras ${ }^{8}$.

A goma guar é uma galactomanana isolada do endosperma da semente de guar, utilizada como suplemento de fibra solúvel ${ }^{9}$. É eficaz na diminuição da hiperglicemia pós-prandial e das concentrações de colesterol, tanto em estudos experimentais ${ }^{10}$ como em indivíduos saudáveis e em diabéticos ${ }^{11}$. O mecanismo de ação da goma guar e de outras fibras solúveis é baseado em sua ação de seqüestrar ácidos biliares no duodeno. Em conseqüência, a excreção fecal de ácidos biliares aumenta nas fezes, diminuindo a quantidade que chega ao fígado pela via entero-hepática. Esse aumento de excreção leva à maior conversão do colesterol hepático em ácidos biliares, reduzindo a concentração intra-hepática de colesterol ${ }^{12}$. Uma das conseqüências da redução do colesterol intracelular é o aumento dos receptores LDL do fígado, aumentado o clearance dessa lipoproteína do sangue ${ }^{12}$. Outros mecanismos também estudados são o aumento da viscosidade intestinal, inibindo diretamente a absorção de colesterol e a sua produção, como resultado da fermentação bacteriana, de ácidos graxos de cadeia curta (como o propionato), que inibiriam a colesterogênese hepática. Embora com efeitos fisiológicos comprovados, essa viscosidade elevada limita o uso da goma guar ou sua incorporação em alimentos.

A ação hipoglicemiante está relacionada à redução na taxa de absorção da glicose alimentar, devido ao aumento da viscosidade do conteúdo intestinal, que retarda o contato da glicose com a área absortiva.

A goma guar parcialmente hidrolisada (GGPH) tem sua viscosidade bastante reduzida, fazendo, assim, com que essa fibra seja facilmente adicionada a alimentos e aceita pelos consumidores. Seu uso como modulador do trânsito intestinal, melhorando tanto a diarréia quanto a constipação intestinal, tem sido comprovado por vários trabalhos ${ }^{13,14}$. Porém, não está claro que, após a perda da viscosidade, todos os efeitos da goma guar (incluindo os efeitos hipocolesterolemiante e hipoglicemiante) sejam mantidos. Por esta razão, o objetivo deste trabalho foi estudar, em camundongos deficientes para o gene do receptor LDL com hiperglicemia induzida por estreptozotocina, se os efeitos metabólicos da goma guar sobre o metabolismo de colesterol e de glicose continuam presentes. 


\section{MÉ T O D OS}

Animais e dieta: foram utilizados 36 camundongos com 6 semanas de idade, homozigotos para a ausência do gene do receptor de LDL $\left(\mathrm{LDLr}^{-/}\right)$gerados no background C57BL/6, obtidos da Jackson Laboratories (EUA) e criados no Biotério do Laboratório de Bioquímica Nutricional, Instituto de Ciências Biológias, Universidade Federal de Minas Gerais (ICB/UFMG). Os animais $L D L r^{-1}$ foram divididos em 4 grupos de 9 animais: camundongos euglicêmicos ou diabéticos alimentados com dieta controle, ou camundongos euglicêmicos ou diabéticos alimentados com dieta controle, suplementados com 10\% goma guar parcialmente hidrolisada durante 4 semanas. Todos os animais $L D L r^{-/}$foram alimentados com dieta aterogênica (Tabela 1).

Os animais tiveram livre acesso à água e foram mantidos em ambiente com ciclo de $12 \mathrm{~h}$ de luminosidade controlada. Durante as quatro semanas experimentais o peso corporal e a ingestão alimentar foram avaliados semanalmente. Foi realizado um controle alimentar entre os grupos controle e GGPH para obter a mesma ingestão

Tabela 1. Composição da dieta aterogênica utilizada para expe-

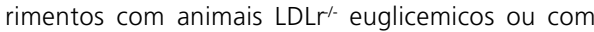
hiperglicemia induzida por estreptozotocina.

\begin{tabular}{lc}
\hline Componente\# & g/kg \\
\hline Fubá & 560 \\
Caseína (99\% proteína) & 145 \\
Celulose & 125 \\
Colina & 10 \\
Mistura mineral* & 50 \\
Mistura vitaminas* & 10 \\
Colesterol & 10 \\
Óleo de soja & 10 \\
Banha de porco & 150 \\
Alfa tocoferol** & 0,04 \\
Ácido cólico** & 5 \\
\hline
\end{tabular}

\#Nas dietas ricas em goma guar parcialmente hidrolisada foram adicionados 75g/kg de ração pronta; *Segundo AIN93-G ${ }^{28}$, confeccionados por Rhoster, SP, Brasil, **Sigma, St Louis, MO, USA. Densidade energética da dieta controle=3,83kcal/g e GGPH (goma guar parcialmente hidrolisada) $=3,86 \mathrm{kcal} / \mathrm{g}$. Percentual protéico $=14,5 \%$, $\mathrm{GGPH}=13,4 \%$. dietética final. No final do experimento, após jejum de 8 a 10 horas, os animais foram anestesiados com Ketamina (130mg/kg) e Xilazina (5,0mg/kg), exangüinados (para dosagens sanguíneas) e mortos por deslocamento cervical com posterior retirada de órgãos. Todos os procedimentos foram aprovados pelo Comitê de Ética Animal da Universidade Federal de Minas Gerais (protocolo n038/03).

O diabetes foi induzido pela injeção intraperitonial de quatro doses de estreptozotocina (80 mg/100g peso), com intervalo de 48 horas cada. A confirmação do Diabetes foi dada pela glicemia acima de $200 \mathrm{mg} / \mathrm{dl}$.

No final do experimento, os camundongos foram anestesiados e amostras de sangue foram coletadas da aorta abdominal. Foram medidos níveis de glicemia de jejum pelo método enzimático, por kit comercial (Katal, Belo Horizonte). Os níveis de colesterol foram medidos de acordo com o método da colesterol oxidase utilizando kit comercial (Katal, Belo Horizonte).

Os fígados dos animais foram removidos, lavados com solução salina, secos em papel filtro e congelados a $-20^{\circ} \mathrm{C}$ até posterior análise (máximo 30 dias). Os lipídeos e o colesterol hepático foram extraídos de acordo com o método de Folch et al. ${ }^{15}$ e a posterior dosagem foi feita pelo kit comercial.

A deposição lipídica nas aortas en face foi detectada utilizando o corante Sudan IV. As aortas foram dissecadas removendo toda a adventícia a partir da válvula aórtica até a bifurcação ilíaca. A árvore aórtica foi aberta longitudinalmente e fixada overnight com solução de formol-sacarose (4,0\% paraformoldeído, $5,0 \%$ de sacarose, $20 \mu \mathrm{mol} / / \mathrm{de}$ BHT, e $2 \mu \mathrm{mol} / / \mathrm{EDTA}, \mathrm{pH} 7,4$ ) a $4^{\circ} \mathrm{C}$. Depois de fixadas, as aortas foram submetidas a uma solução de $70,0 \%$ de etanol durante 5 minutos, posteriormente foram coradas por 10 minutos sob agitação, numa solução filtrada contendo 0,5\% de Sudan IV, 35,0\% de etanol e 50,0\% de acetona e descoradas por 5 minutos em solução de $80,0 \%$ de etanol. As imagens das aortas coradas com Sudan IV foram capturadas por scanner, conge- 
ladas e medidas utilizando-se programa analisador de imagens KS300. Os cálculos das lesões das aortas foram feitos como porcentagem da lesão em relação à área total da aorta. O valor médio por grupo foi utilizado para comparações estatísticas.

A comparação dos dados obtidos entre os grupos LDLr-/ controles e suplementados com GGPH, diabéticos ou euglicêmicos foi feita utilizando-se análise de variância e correção de borrefoni, calculado pelo programa Sigma Plot (Scientific Graphing Software, Jandel Corporation). Foi considerado um nível de significância de 5\%.

\section{RESULTADOS}

Os resultados obtidos com animais $L D L r^{-1-}$ mostram que não houve diferenças estatísticas no consumo alimentar entre os grupos controle e suplementado com GGPH. Porém, animais diabéticos apresentaram um consumo maior de ração, comparados aos não diabéticos (Tabela 2).

Em relação à evolução ponderal, não houve diferenças estatísticas entre os grupos (Tabela 2). Estes resultados são esperados devido à hiperfagia característica de animais com redução da concentração de insulina.

Quanto ao colesterol sérico, nota-se que a ingestão de goma guar parcialmente hidrolisada não promoveu a sua redução. Houve um aumento na colesterolemia dos animais euglicêmicos recebendo o hidrolisado, em relação aos controles. Essa mesma tendência foi vista nos animais diabéticos, porém sem significância estatística (Figura 1).

A goma guar parcialmente hidrolisada também aumentou a concentração hepática de colesterol em animais euglicêmicos. Essas alterações não foram notadas em animais diabéticos, como também os teores de lipídeos hepáticos totais (Tabela 2). Embora modificações no metabolismo de colesterol tenham sido estatisticamente diferentes nos grupos de animais euglicêmicos, não houve diferenças significantes na área da aorta torácica e abdominal afetada por lesões ateroscleróticas nesses animais. Em animais diabéticos, por outro lado, houve uma redução estatisticamente significante da área afetada, sugerindo um efeito benéfico do hidrolisado nesses animais (Figuras 2 e 3 ).

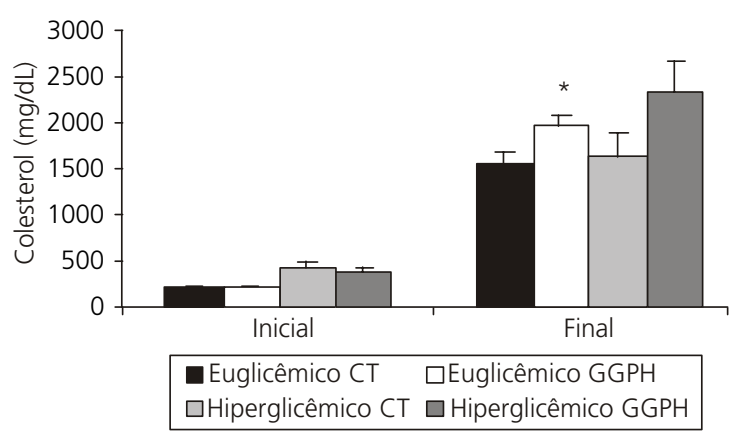

Figura 1. Colesterolemia inicial (TO) e final (T4) de camundongos LDLr- euglicêmicos ou hiperglicêmicos recebendo dieta controle (CT) ou contendo $7,5 \%$ de goma guar parcialmente hidrolisada (GGPH) por 4 semanas.

${ }^{*} p<0,05$ em relação ao grupo CT euglicêmico na mesma semana.

Tabela 2. Consumo alimentar, evolução ponderal e lipídeos hepáticos de animais LDLr- euglicemicos ou com hiperglicemia induzida por estreptozotocina suplementados ou não com 7,5\% de goma guar parcialmente hidrolisada (GGPH).

\begin{tabular}{|c|c|c|c|c|c|c|c|c|}
\hline \multirow[b]{3}{*}{ Consumo alimentar (g/animal/semana) } & \multicolumn{4}{|c|}{ Euglicêmico } & \multicolumn{4}{|c|}{ Hiperglicêmico } \\
\hline & \multicolumn{2}{|c|}{ Controle } & \multicolumn{2}{|c|}{ GGPH } & \multicolumn{2}{|c|}{ Controle } & \multicolumn{2}{|c|}{ GGPH } \\
\hline & 14,7 & $(1,5)$ & 14,5 & $(1,6)$ & 33,8 & $(9,8)$ & 32,9 & $(7,5)$ \\
\hline Peso (g) & 15,4 & $(1,2)$ & 16,1 & $(0,6)$ & 16,8 & $(0,8)$ & 16,8 & $(0,6)$ \\
\hline Final & 13,6 & $(1,0)$ & 15,2 & $(0,5)$ & 15,2 & $(0,9)$ & 15,9 & $(1,1)$ \\
\hline Lipídeos hepáticos (mg/g tecido) & 125,0 & $(10,8)$ & 158,0 & $(85,0)$ & 142,0 & $(12,0)$ & 158,0 & $(5,0)$ \\
\hline Colesterol hepático (mg/g tecido) & 16,2 & $(1,4)$ & 23,6 & $(1,5)^{*}$ & 23,8 & $(3,3)$ & 25,8 & $(1,4)$ \\
\hline
\end{tabular}

* $p<0,05$ entre controle e GGPH do mesmo grupo. Teste " $t$ " de student. 


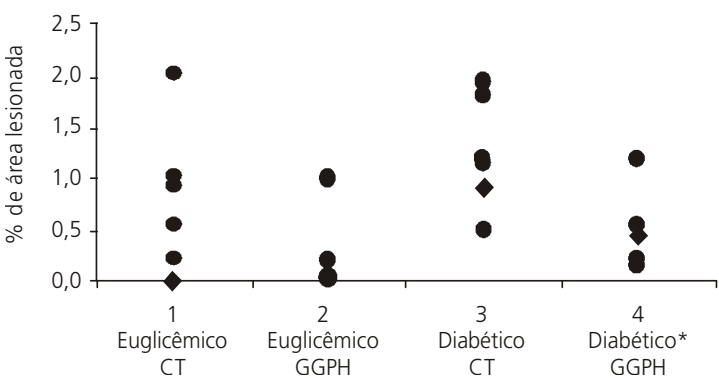

Figura 2. Percentagem de lesão em aorta torácica/abdominal de camundongos LDLr- euglicêmicos e hiperglicêmicos ( $n=6$ por grupo), recebendo dieta controle ou suplementada com 7,5\% de goma guar parcialmente hidrolisada (GGPH) por 4 semanas. Coloração SUDAN IV, que cora em vermelho especificamente lipídeos.

${ }^{\star} p<0,05$ em relação ao grupo $C T$ diabético

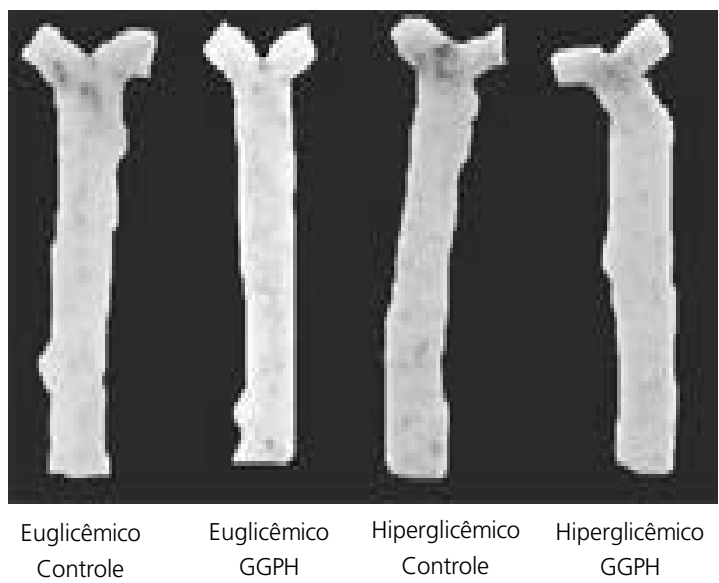

Figura 3. Aorta torácica/abdominal de camundongos LDLr ${ }^{-/}$ euglicêmicos e hiperglicêmicos, recebendo dieta controle ou suplementada com 7,5\% de goma guar parcialmente hidrolisada (GGPH) por 4 semanas. Coloração SUDAN IV, que cora em vermelho especificamente lipídeos.

\section{DIS C U S S Ã O}

Os efeitos mais relevantes da goma guar parcialmente hidrolisada (GGPH) estão ligados à sua capacidade de sofrer fermentação bacteriana e modular a microbiota intestinal, sendo sua administração benéfica em casos de hipotrofia intestinal, diarréia, entre outros ${ }^{9}$. Alguns trabalhos indicam que o ácido graxo de cadeia curta, ácido propiônico, produto da fermentação bacteriana de fibras no intestino ${ }^{16}$, tem efeito hipocolesterolêmico por reduzir a colesterogênese hepática. Estudos anteriores deste grupo de pesquisadores ${ }^{17,18}$, utilizando goma guar não hidrolisada administrada a ratos isentos de germes (nos quais não ocorre fermentação intestinal) ou heteroxênicos (colonizados com microbiota humana) não reforçam esta hipótese. Os resultados desses estudos mostraram que a ação da goma guar em reduzir o colesterol plasmático foi maior em animais isentos de germe (sem a produção de ácido propiônico) que nos animais heteroxênicos. Além disso, não houve diferenças na colesterogênese hepática ${ }^{17} \mathrm{com}$ o uso dessa fibra, como o visto em estudos com acetato $\mathrm{C}^{14}$.

Embora estudos sugiram que a goma guar parcialmente hidrolisada reduz a velocidade de absorção de lipídeos no intestino, por reduzir a emulsificação pelos ácidos biliares ${ }^{19,20}$, o efeito em longo prazo de GGPH ainda não está confirmado na literatura ${ }^{9}$. Um estudo realizado em ratos jovens ${ }^{21}$ mostrou a redução do colesterol e triacilgliceróis sanguíneos e hepáticos, quando alimentados com dieta padrão e $6,0 \%$ de GGPH. Entretanto, esses efeitos não foram vistos em ratos de oito meses de vida ${ }^{22}$. Favier et al. ${ }^{23}$, estudando o metabolismo de colesterol em ratos alimentados com dieta contendo $0,4 \%$ de colesterol e suplementada com goma guar altamente viscosa ou GGPH, encontraram menor colesterol sérico, maior excreção fecal de esteróides com balanço negativo de colesterol com o uso de goma guar. A goma guar parcialmente hidrolisada teve efeitos nulos na colesterolemia e um balanço ligeiramente positivo de colesterol no organismo. Os resultados deste estudo estão em desacordo com os descritos acima, pois houve um aumento tanto no colesterol plasmático quanto hepático em camundongos euglicêmicos suplementados com GGPH. Essas diferenças podem ser devidas aos diferentes delineamentos experimentais. Yamada et al. ${ }^{21}$ utilizaram ratos alimentados com dieta padrão, enquanto neste estudo foram utilizados camundongos que desenvolvem hipercolesterolemia e placas ateroscleróticas, consumindo dietas atero- 
gênicas. Favier et al..$^{23}$, por sua vez, utilizaram $0,4 \%$ de colesterol enquanto este estudo utilizou mais que o dobro $(1,0 \%)$ de colesterol, além de $0,5 \%$ de ácido cólico. As particularidades no perfil de lipoproteínas e no metabolismo de colesterol podem ter levado aos diferentes resultados. Talvez, o efeito na redução da emulsificação lipídica possa ter sido superado pela presença de colesterol e de ácidos biliares na dieta estudada. Igualmente, a maior quantidade de colesterol e ácidos biliares na dieta pode ter induzido a um balanço mais positivo, como sugerido pela intensa hipercolesterolemia, reduzindo as possíveis diferenças que a GGPH causaria na absorção intestinal. Em adição, produtos do metabolismo da GGPH no fígado podem estar, de alguma forma, aumentando o clearance ou reduzindo a exportação de lipoproteínas nesses camundongos.

Embora alguns estudos citados acima mostrem efeitos da GGPH nos lipídeos hepáticos e plasmáticos, os resultados podem apenas levar à sugestão de seu possível efeito na aterosclerose. Este parece ser o primeiro trabalho mostrando os efeitos de goma guar parcialmente hidrolisada diretamente na formação de placa aterosclerótica.

Os relatos do efeito da GGPH no metabolismo lipídico de diabéticos são ainda mais escassos e não há estudos associando GGPH, Diabetes e desenvolvimento de aterosclerose. Os resultados desta investigação mostram que o uso de GGPH pode retardar o desenvolvimento da placa em animais hiperglicêmicos, o que traria benefícios adicionais com o uso da fibra nessa doença.

Independentemente dessas alterações no metabolismo de lipídeos, camundongos diabéticos tiveram redução estatisticamente significante na glicemia de jejum. A fermentação de goma guar nativa ou hidrolisada produz quantidades aumentadas de ácido butírico, e ambos (fibra e butirato) são capazes de reduzir a glicemia em ratos $^{24}$. Os resultados deste estudo estão de acordo com os da literatura, mostrando a redução da glicemia com o uso de GGPH. A redução da glicemia está associada à redução da aterosclerose em animais hiperglicêmicos em uso de GGHP. Nos animais euglicêmicos não houve diferenças entre o tamanho de placas entre os grupos controle e $\mathrm{GGPH}$. Os resultados sugerem que a GGPH afeta a aterogênese por mecanismos relacionados ao Diabetes em si, e não apenas à colesterolemia, uma vez que os níveis de colesterol não foram diferentes entre os grupos GGPH euglicêmico e hiperglicêmico ao final do experimento (Figura 1).

O Diabetes Mellitus evolui com o aparecimento de vários fatores contribuintes para a aterosclerose, como: aumento dos ácidos graxos livres e das lipoproteínas aterogênicas e redução da fração protetora HDL; aumento da oxidação de LDL pelos produtos finais de glicação; e alterações no endotélio vascular em decorrência da hiperglicemia 25 .

A estreptozotocina, agente indutor do Diabetes utilizado neste estudo, causa uma inflamação nas ilhotas de Langerhans e superprodução de radicais superóxido e óxido nítrico, sendo este último citotóxico para as células $\beta$ do pâncreas ${ }^{26}$. É bem conhecido que processos inflamatórios e estresse oxidativo aceleram a aterosclerose, tanto pela maior produção de citocina como pela ativação de linfócitos $T$, ambos envolvidos na produção de moléculas de adesão e na maior migração de leucócitos plasmáticos para o subendotélio de artérias, e ainda pela ativação de componentes trombogênicos ${ }^{27}$. Como dito acima, a GGPH reduziu as lesões apenas em animais $L D L r^{-1}$ diabéticos, mas não em animais euglicêmicos. Estes resultados sugerem que os mecanismos de tal efeito devem estar associados à hiperglicemia e ao estresse oxidativo, induzidos pela estreptozotocina, e não apenas à hipercolesterolemia.

Assim, os resultados neste estudo mostram que a goma guar parcialmente hidrolisada pode reduzir a aterosclerose associada ao Diabetes Mellitus.

\section{A GRADECIMENTOS}

Os autores agradecem à Katal, pela doação dos kits; a Maria Helena Alves de Oliveira, pela 
assistência aos camundongos LDLr ${ }^{-/}$, ao Conselho Nacional de Desenvolvimento Científico e Tecnológico (CNPq), pelas bolsas de Iniciação Científica, e produtividade em pesquisa concedidas a LRF e JIAL, respectivamente, e à Coordenação de Aperfeiçoamento de Pessoal de Nível Superior (CAPES), pela bolsa de doutorado de LRP.

\section{REFERÊ N CIAS}

1. National Cholesterol Education Program. Third report of the expert panel on detection, evaluation, and treatament of high blood cholesterol in adults. JAMA. 2001; 285:2496-9.

2. Juhan-Vague I, Alessi MC, Vague P. Increased plasma plasminogen activator inhibitor 1 levels. A possible link between insulin resistance and atherothrombosis. Diabetologia. 1991; 34(7): 457-62.

3. Izkhakov E, Meltzer E, Rubinstein A. Pathogenesis and management of diabetic dyslipidemia. Treat Endocrinol. 2003; 2(4):231-45.

4. Large V, Peroni O, Letexier D, Ray H, Beylot M. Metabolism of lipids in human white adipocyte. Diabetes Metab. 2004; 30(4):294-309.

5. Ishibashi S, Goldstein JL, Brown MS, Herz J, Burns DK. Massive xanthomatosis and atherosclerosis in cholesterol-fed low density lipoprotein receptor-negative mice. J Clin Invest. 1994; 93(5): 1885-93.

6. Lusis AJ. Atherosclerosis. Nature. 2000; 407(6801): 233-41.

7. Cascieri MA. The potential for novel anti-inflammatory therapies for coronary artery disease. Nat Rev Drug Discov. 2002; 1(2):122-30.

8. Press NA. Dietary reference intakes: proposed definition of dietary fiber. Washington: National Academy Press; 2001.

9. Slavin JL, Greenberg NA. Partially hydrolyzed guar gum: clinical nutrition uses. Nutrition. 2003; 19(6): 549-52.

10. Stanogias G, Pearce GR. The digestion of fibre by pigs. 3. Effects of the amount and type of fibre on physical characteristics of segments of the gastrointestinal tract. Br J Nutr. 1985; 53(3): 537-48.

11. Kishida T, Nogami H, Ogawa H, Ebihara K. The hypocholesterolemic effect of high amylose cornstarch in rats is mediated by an enlarged bile acid pool and increased fecal bile acid excretion, not by cecal fermented products. J Nutr. 2002; 132(9):2519-24.

12. Farmer JA, Gotto AM, Jr. Currently available hypolipidaemic drugs and future therapeutic developments. Baillieres Clin Endocrinol Metab. 1995; 9(4):825-47.

13. Alam NH, Meier R, Schneider $H$, Sarker SA, Bardhan PK, Mahalanabis D, et al. Partially hydrolyzed guar gum-supplemented oral rehydration solution in the treatment of acute diarrhea in children. J Pediatr Gastroenterol Nutr. 2000; 31(5):503-7.

14. Spapen H, Diltoer M, van Malderen C, Opdenacker $G$, Suys E, Huyghens L. Soluble fiber reduces the incidence of diarrhea in septic patients receiving total enteral nutrition: a prospective, double-blind, randomized, and controlled trial. Clin Nutr. 2001; 20(4):301-5.

15. Folch J, Lees GH, Sloane-Stanley GH. A simple method for the isolation and purification of total lipids from animal tissues. J Biol Chem. 1957; 226(1):497-509.

16. Cheng $\mathrm{HH}$, Lai $\mathrm{MH}$. Fermentation of resistant rice starch produces propionate reducing serum and hepatic cholesterol in rats. J Nutr. 2000; 130(8):1991-5.

17. Alvarez-Leite J, Andrieux C, Ferrezou J, Riottot M, Vieira E. Evidences for absence of participation of the microbial flora in the hypocholesterolemic effect of guar gum in gnotobiotic rats. Comp Biochem Physiol. 1994; 109(2):503-10.

18. Alvarez-Leite JI, Andrieux C, Ferezou J, Riottot M, Vieira EC. Heat or gamma irradiation sterilization affects the hypocholesterolemic effect of guar gum in axenic and heteroxenic rats. Braz J Med Biol Res. 1994; 27(3):677-89.

19. Kondo S, Xiao J, Takahashi N, Miyaji K, Iwatsuki K, Kokubo S. Suppressive effects of dietary fiber in yogurt on the postprandial serum lipid levels in healthy adult male volunteers. Biosci Biotechnol Biochem. 2004; 68(5):1135-8.

20. Minekus M, Jelier M, Xiao J, Kondo S, Iwatsuki K, Kokubo $S$, et al. Effect of partially hydrolyzed guar gum (PHGG) on the bioaccessibility of fat and cholesterol. Biosci Biotechnol Biochem. 2005; 69(5):932-8.

21. Yamada K, Tokunaga Y, Ikeda A, Ohkura K, Mamiya $S$, Kaku S, et al. Dietary effect of guar gum and its partially hydrolyzed product on the lipid metabolism and immune function of SpragueDawley rats. Biosci Biotechnol Biochem. 1999; 63(12):2163-7.

22. Yamada K, Tokunaga Y, Ikeda A, Ohkura K, Kaku-Ohkura S, Mamiya S, et al. Effect of dietary fiber on the lipid metabolism and immune 
function of aged Sprague-Dawley rats. Biosci Biotechnol Biochem. 2003; 67(2):429-33.

23. Favier M, Bost $P$, Guittard C, Demigne C, Remesy $C$. The cholesterol-lowering effect of guar gum is not the result of a simple diversion of bile acids toward fecal excretion. Lipids. 1997; 32(9): 953-9.

24. Weaver G, Tangel C, Krause J, Alpern H, Jenkins $P$, Parfitt $M$, et al. Dietary guar gum alters colonic microbial fermentation in azoxymethane-treated rats. J Nutr. 1996; 126(8):1979-91.

25. Brownlee M. The pathobiology of diabetic complications: a unifying mechanism. Diabetes. 2005; 54(6):1615-25.
26. Szkudelski T. The mechanism of alloxan and streptozotocin action in B cells of the rat pancreas. Physiol Res. 2001; 50(6):537-46.

27. Hansson GK, Libby P, Schonbeck U, Yan ZQ. Innate and adaptive immunity in the pathogenesis of atherosclerosis. Circ Res. 2002; 91(4):281-91.

28. Reeves PG, Nielsen FH, Fahey GCJ. AIN-93 purified diets for laboratory rodents: final report of the American Institute of Nutrition ad hoc writing committee on the reformulation of the AIN-76A rodent diet. J Nutr. 1993; 123(11):1939-51.

Recebido em: 13/9/2005

Versão final reapresentada em: 18/1/2006 Aprovado em: 21/6/2006 